\title{
Analysis of Infrared Radiation Characteristics and Penetration Measures of Warhead
}

\author{
Xiao Liu, Yehui Li \\ Branch 30, Unit 91049, Qingdao, China \\ Email: 826503853@qq.com
}

How to cite this paper: $\mathrm{Liu}, \mathrm{X}$. and $\mathrm{Li}$, Y.H. (2018) Analysis of Infrared Radiation Characteristics and Penetration Measures of Warhead. Journal of Computer and Communications, 6, 162-170. https://doi.org/10.4236/jcc.2018.611016

Received: November 5, 2018

Accepted: November 12, 2018

Published: November 19, 2018

\begin{abstract}
Ballistic target recognition occupies a unique and important position in many application fields of target recognition because of its challenge and important position of ballistic missile defense in national security; recognition time of defense system becomes very limited because of ballistic missile high-speed flight; recognition distance of defense system is also due to stealth technology. The integrated application of active jamming and passive decoy greatly increases the difficulty of identification of defense system. Because of its special status and challenge, ballistic target recognition has attracted wide attention of researchers at home and abroad, making it one of the most important issues in infrared target recognition research at home and abroad. In this paper, the infrared characteristics of a ballistic missile warhead target/decoy are analyzed, and the corresponding penetration measures are put forward according to the analysis results.
\end{abstract}

\section{Keywords}

Ballistic Missile, Warhead, Infrared Radiation, Penetration Measures

\section{Introduction}

In order to improve the penetration capability of ballistic warhead, a large number of sub-warheads are formed in the middle of the ballistic missile by means of warheads miniaturization and divergent multi-warheads technology, which saturates the identification and fire control capability of the defense system [1]. Among them, the guided multi warheads can not only include biochemical warheads, but also nuclear warheads. In addition to forming complex target groups in the middle, the penetrating party can also use a variety of means to reduce the detectability of warhead itself. For example, the use of warhead stealth technology greatly reduces the detection distance of warhead [2] [3]. In addition, the 
penetration side can also wrap the reentry vehicle in the metal balloon. With the above countermeasures, the difficulty of target recognition is greatly increased.

In addition to obtaining the information of warhead target, it also obtains the information of decoy, stars and noise of detection system, which will interfere with the detection and recognition of target. In some aspects, the similarity with the target is interference, while the detection and recognition of the target is based on the difference between the target and the interference factors in some aspects [4]. When the target occupies only a few pixels in the field of view of the detection system, the target information can be obtained very little, only time and space information. Temporal information is the change of target brightness with time, while spatial information is the change of target motion trajectory. It is very necessary to analyze the infrared radiation characteristics of the warhead target/background in the middle of the trajectory. The basis of detecting and identifying the target is to clarify the difference between the target and the decoy.

Usually, a threshold is set in background removal. If the pixel is smaller than the threshold, it is considered as the background. If the threshold is larger than the threshold, it is considered as the target. Threshold setting methods include optimal threshold and local optimal threshold. Another method uses the background modeling method to estimate the mean and standard deviation of the background, set a global threshold, and use the statistics of the neighborhood of the point to judge whether the background is or not [5]. It is essentially a transformation of the background and the target probability density function, making it more conducive to highlighting the target and suppressing noise. In this paper, a new algorithm based on small area filtering is proposed, which uses global threshold. To some extent, the algorithm is not sensitive to threshold. Under the same threshold, it can reduce $70 \%$ - $80 \%$ noise points compared with direct threshold segmentation.

\section{Warhead Target/Decoy Thermal Analysis}

Taking the warhead target in the middle of the trajectory as an example, the heat source on the target surface includes internal heat source and external heat source. Internal heat sources are all the equipment enclosed in the warhead target case, which emit part of the heat generated by work, and heat the target from the inside. External heat sources refer to all objects that exist outside warhead in space. In the middle of the ballistic trajectory of a ballistic missile, because there is no instrument or equipment to heat the warhead, the internal heat source for heating the warhead surface can be neglected. While the ballistic missile trajectory is near ground flight, the main external heat sources include the earth and the sun. They heat the warhead from the surface of the warhead/bait. There are three main ways of heating:

1) The sun irradiated warhead directly;

2) Earth directly irradiated warhead;

3) Sunlight reflected from the earth illuminated warhead. 
It can be seen that the heating effect of the external heat source is not only related to the surface structure and thermos-physical properties of warhead (absorptivity, emissivity, thermal conductivity, specific heat capacity and density, etc.), but also related to the shape and attitude of warhead and the relative space position between the sun, the earth and warhead [6]. When warhead is in the solar irradiation area, the solar energy can directly irradiate the outer surface of warhead, while at night, it is in the shadow area of the earth, the sun can not directly irradiate the surface of warhead, so the infrared radiation characteristics of warhead in different space positions will be quite different. The schematic diagram of the heat balance of the warhead in the middle of the ballistic trajectory is shown in Figure 1.

\section{Warhead Target/Decoy External Surface Temperature Field Calculation}

Thermal network analysis often analyzes and calculates the inner surface panel and the inner instrument node of the space target, because the sensor can only detect the radiation emitted from the outer surface of the space target, so only from the infrared simulation point of view, the temperature information of the inner surface panel of the space target is useless. The infrared characteristic model of warhead target/decoy is established.

The ideal principle of panel partitioning is that the isothermal and properties in the panel are uniform, but the temperature distribution is unknown. Therefore, we must find the principle of partition of faces which is equivalent to ideal principles. According to the geometric shape and orbit of warhead, the surface heating of warhead can be preliminarily analyzed, which can be used as the basis for the partition of the panel. The heating and temperature change greatly in the vicinity of the region where the geometric shape changes abruptly, so it is necessary to divide the region more carefully. On the contrary, the geometric shape changes less, the heating changes less, the temperature distribution is relatively uniformed, and the larger panel can be selected. The normal vector at the center of the panel represents the normal vector of the panel. This division ensures the accuracy of the result and the computation requirement [7].

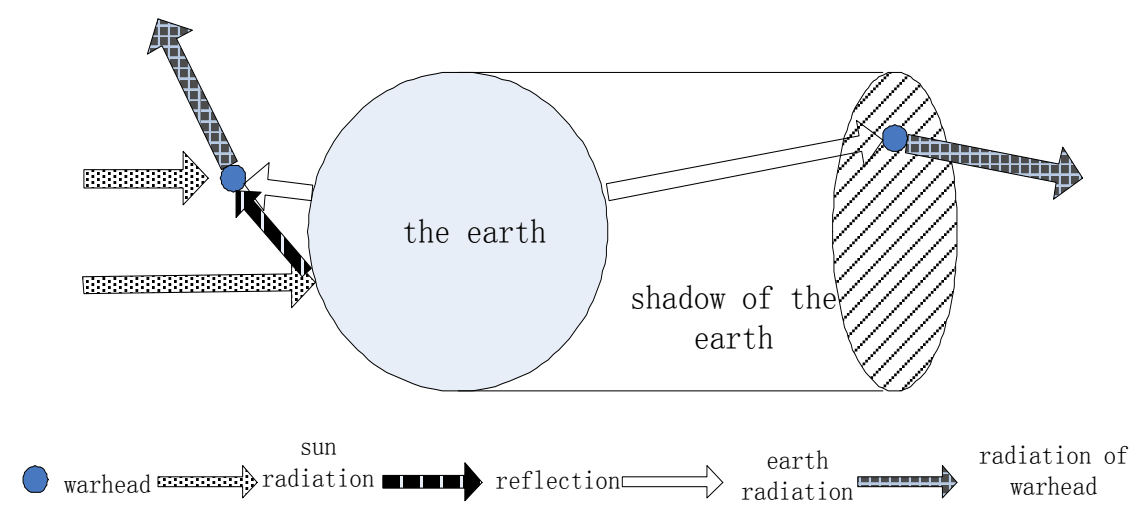

Figure 1. Schematic diagram of heat balance of warhead in Midcourse of trajectory. 
The three main aspects of the thermal energy of a single panel include: the thermal energy of the incident panel, the thermal energy of the panel radiation and the thermal energy stored or released by the panel due to temperature changes. For the thermal energy of the incident panel, not only the radiation heating of the environment, but also the heat exchange between the panel and the inner and outer surfaces of the same panel should be considered. Suppose the partition element as follows: the area of the facets $j$ is $A_{p}$ the temperature is $T_{j}$

Direct radiation heating of solar panel:

$$
Q_{1 j}=A_{j} a_{S j} E_{s u n}\left(\frac{R_{S E}}{R_{S T}}\right)^{2} \varphi_{1 j}
$$

In the above formula, $a_{S j}$ is the coefficient of solar radiation absorbed by a panel $j ; R_{S E}$ is the average distance between the sun and the earth, whose value is $1.49985 \times 10^{8} \mathrm{~km} ; R_{S T}$ is the distance between the sun and the warhead target/decoy; $\varphi_{1 j}$ is the solar radiation angle coefficient of a panel $j$, indicating the proportion of solar radiation reaching the panel $j ; E_{\text {sun }}$ is the solar constant outside the atmosphere, which means that the distance between the earth and the sun is the average distance between the sun and the earth. At a distance, the total irradiance produced by the sun outside the earth's atmosphere, which is generally expressed by $E_{\text {sun }}=1353 \mathrm{~W} / \mathrm{m}^{2}$.

The biggest external heat source of warhead target/bait is the sun. If we observe from the earth, the sun occupies only a half solid angle. Therefore, the solar radiation at the magnitude of solar terrestrial distance can be treated as a point source. The relative volume of the warhead target/decoy is very small at this distance, so the sunlight projected onto the surface of the warhead target/decoy can be regarded as parallel light.

Because of the different materials, the material density, specific heat and panel thickness of decoy and warhead target are quite different. The difference of infrared characteristics between warhead target and decoy is mainly caused by the combined action of harmony $Q_{c j}, Q_{5 j}$ and $Q_{6 j}$.

\section{Analysis of Infrared Radiation Characteristics of Warhead Target and Balloon Bait}

Loading warhead into the metal balloon bait is the most mature anti simulation bait technology at present. In the middle of the trajectory, a balloon equipped with warhead will be released with a large number of empty balloon bait. Because the radar detection beam cannot penetrate the thin metal coating of the balloon, the radar cannot recognize the object inside the balloon. However, warhead emits heat, which conducts heat from the outside balloon, causing a temperature difference between the balloon with warhead and the balloon bait with the inside hollow. Thus, the infrared detector of the ballistic missile defense system will recognize the true warhead according to the temperature difference between them. Therefore, this paper takes the anti-simulated balloon decoy as a 
typical research object, and studies the infrared radiation characteristics in the middle of its trajectory, which has certain theoretical significance and application value.

In this paper, the thermal characteristics of balloon decoys and balloons equipped with warhead under two different conditions of day attack and night attack are analyzed theoretically, the thermal equilibrium temperature of balloon decoys is calculated, the time needed to reach thermal equilibrium is calculated, and the temperature variation trend of balloon equipped with warhead is analyzed quantitatively. Aiming at the temperature difference between the balloon decoy and the balloon equipped with warhead, this paper puts forward some measures to eliminate the difference, which can reduce the probability of the true warhead being identified by the infrared detector.

\subsection{Infrared Radiation Characteristics of Balloon Bait}

1) Thermal equilibrium temperature of balloon bait

Because the middle part of the ballistic trajectory is in a nearly vacuum space, there is no heat conduction and convective heat transfer except thermal (infrared) radiation.

a) Balloons decoy during the daytime attack

It is mainly determined by the surface coating to determine the target equilibrium temperature under sunlight. The attacker can easily change the equilibrium temperature of the balloon by using various surface coatings, such as coatings. Table 1 lists the equilibrium temperatures of spherical targets using different surface coatings under daylight exposure. The equilibrium temperatures of various balloons vary over $300 \mathrm{~K}$, the equilibrium temperatures of white titanium dioxide paint are about $227 \mathrm{~K}$ and polished gold foil is about $540 \mathrm{~K}$.

Table 1. Visible light absorptivity, infrared emissivity and equilibrium temperature of various materials.

\begin{tabular}{ccccc}
\hline Surface coating & $\alpha$ & $\varepsilon$ & $\frac{\alpha}{\varepsilon}$ & $\begin{array}{c}\text { The balance } \\
\text { temperature of balloon } \\
\text { bait during daytime (K) }\end{array}$ \\
\hline White $\mathrm{TiO}_{2}$ coatings & 0.19 & 0.94 & 0.20 & 226.5 \\
White epoxy coatings & 0.248 & 0.924 & 0.27 & 236.9 \\
White enamel & 0.252 & 0.853 & 0.30 & 240.8 \\
Polyester film & 0.17 & 0.5 & 0.34 & 246.8 \\
Aluminum silicon coating & 0.25 & 0.28 & 0.89 & 299.2 \\
Grey TiO & 0.87 & 0.87 & 1.00 & 306.7 \\
Black paint & 0.975 & 0.874 & 1.12 & 314.3 \\
Aluminum based coatings & 0.54 & 0.45 & 1.20 & 319.4 \\
Aquadag coatings & 0.782 & 0.49 & 1.60 & 340.9 \\
Aluminum foil & 0.192 & 0.036 & 5.33 & 454.4 \\
Polishing gold coating & 0.301 & 0.028 & 10.8 & 539.7
\end{tabular}

Note: The solar absorptivity of the surface materials of the balloon bait $\alpha$ and the infrared radiation of the surface materials of the balloon bait $\varepsilon$ are shown in the table. 
Table 1 shows that using one or more paints to coat the entire balloon or part of the balloon with aluminum foil on the outer surface, the balloon can reach the equilibrium temperature of $227-454 \mathrm{~K}$. For example, if the aluminum outer layer is completely covered with white titanium dioxide, the balloon can achieve a balance temperature of $227 \mathrm{~K}$. If the surface of the balloon is partially covered with black paint, the balloon can reach a balance temperature between 314 and $454 \mathrm{~K}$, and the final temperature depends on how many surfaces are painted with black paint. Therefore, for each balloon, the offense can choose different equilibrium temperatures.

b) Night attack balloon bait

Night attacks are very different from day attacks because the only major external source of heat at night is infrared radiation from the Earth. At night, the equilibrium temperature of a spherical balloon in LEO will remain around 180K, depending on the surface composition of each balloon.

At such a low equilibrium temperature, the chamber temperature warhead inside the balloon has great influence. For example, if there is no warhead in the balloon, the equilibrium temperature is $299.2 \mathrm{~K}$ in the sun and $187 \mathrm{~K}$ at night. Even if there is warhead in the balloon, the surface emissivity of the warhead is 0.036 . The heat exchange is only caused by the radiation of the warhead, and its equilibrium temperature is only $187 \mathrm{~K}$ (if the heat transfer of the warhead is heat). When radiation is 5 times, the equilibrium temperature can reach $204 \mathrm{~K}$. Even so, the attacker can take direct countermeasures to prevent the defense system from recognizing targets based on the thermal effects of the warhead in the balloon.

2) The time needed to achieve equilibrium temperature

Taking the balloon model adopted by the US military in space experiment as an example, this paper illustrates the time-dependent law of the equilibrium temperature of the balloon decoy. In the space test, the USArmy used a double ball with a diameter of $3 \mathrm{M}$. In the experiment, the balloon bait used two schemes, one is the thin material balloon bait, the outer layer is $0.00025 \mathrm{~cm}$ thick aluminum foil, the inner layer is $0.00064 \mathrm{~cm}$ thick polyester film layer; the other is the material thick balloon bait, the outer layer is $0.0025 \mathrm{~cm}$ thick aluminum foil, the inner layer is $0.0025 \mathrm{~cm}$ thick polyester film layer.

Assuming that the initial surface temperature of the balloon decoy is $300 \mathrm{~K}$, the fourth-order Runge-Kutta method is used to plot the curves of the daytime balloon decoy temperature with time.

The thin balloon bait takes about 1 - 2 minutes to reach equilibrium at room temperature $(300 \mathrm{~K})$. In the same case, for a thick material balloon bait, it takes a few minutes to ten minutes. If the initial temperature of the balloon bait is very different from the equilibrium temperature during the daytime attack, the temperature of the balloon can rise or fall rapidly to the equilibrium temperature because of the very low heat capacity of the balloon. How long the process takes depends on the difference between the initial and equilibrium temperatures of the balloon and the thermal capacity of the balloon. 
During the night attack, the balloon bait with thin material reaches the equilibrium temperature of $180 \mathrm{~K}$ in about 2 minutes, and the balloon bait with thick material reaches the equilibrium temperature in more than 10 minutes, which has nothing to do with the surface coating.

\subsection{Infrared Radiation Characteristics of Balloon Equipped with Warhead}

Compared with the interior hollow balloon bait, warhead has heavy mass and large heat capacity, and takes several hours to reach the equilibrium temperature. So after the balloon and warhead are put in the outer space, the warhead placed in the balloon will affect the thermal performance of the balloon. Warhead transfers heat to the balloon (or balloon transfers heat to warhead) in four ways: heat radiation, heat conduction through the support of the balloon that fixes the warhead, heat conduction through the gas in the balloon, and gas convection caused by movement. Convection is the main factor affecting the change of balloon temperature, which is caused by heat radiation and movement.

During the daytime attack, the balloon's equilibrium temperature depends on the surface coating. The temperature of the warhead inside the balloon rises or falls rapidly. The warhead inside the balloon changes very slowly. The warhead and the balloon will change the balloon's temperature to the initial temperature by four heat transfer modes. Night attacks are very different from day attacks because the only external source of heat at night is infrared radiation from the Earth. In the evening, when the balloon bait is on the near earth orbit, its equilibrium temperature will remain at about $180 \mathrm{~K}$. At such a low equilibrium temperature, the influence of warhead on the balloon room temperature is very large, its equilibrium temperature can only reach about $200 \mathrm{~K}$.

\section{Penetration Measures Analysis}

From the above analysis, it can be seen that the thermal characteristics of the balloon decoy and the balloon with warhead are different after the launch. If the measures are not taken properly, the infrared detector will easily distinguish the target. Next, we analyze the 2 cases from day attack and night attack.

\subsection{Daytime Attack}

During the daytime attack, the main difference in the thermal characteristics between the balloon decoy and the balloon with warhead is that the temperature of the balloon decoy will rise rapidly or fall to the equilibrium temperature, which depends on the surface coating of the decoy, and the temperature of the balloon with warhead will rise or fall to the initial temperature at the time of release.

If the equilibrium temperature of the balloon bait is very different from the initial temperature of the bait, the infrared detector will easily identify the warhead target. Therefore, for daytime attack, the balloon decoy can be balanced at about the same temperature as the initial temperature of nuclear warhead by se- 
lecting the appropriate surface coating of balloon decoy, thus basically eliminating the various thermal effects that nuclear warhead may have on the balloon. For example, if the initial temperature of warhead is close to room temperature $(300 \mathrm{~K})$, the balloon surface can be coated with aluminum-silicon coating, making the balloon temperature change in a small range around $300 \mathrm{~K}$. In this way, all infrared detectors of the ballistic missile defense system (including those on the LEO SBIRS satellite and interceptor) can be prevented from identifying balloon and balloon decoys with nuclear warhead.

\subsection{Night Attack}

When attacking at night, the equilibrium temperature of the balloon with warhead is about $20 \mathrm{~K}$ higher than that of the balloon decoy. In order to prevent the infrared detector from detecting warhead, two countermeasures, active and passive, can be taken to solve this problem.

Active measures: the use of small battery-powered heater balloon bait temperature increased to the balloon with warhead equivalent to the temperature. Passive measures: the first method is to use super insulating or low-radiation materials, such as bright aluminum foil or polished silver coating, to cover the nuclear warhead to reduce the heat transfer of warhead to the balloon; the second method is to use balloons of different shapes, so that all balloons have different equilibrium temperatures, which vary only a few degrees. Among the balloons, an infrared detector with a nuclear warhead and a ballistic missile defense system is also unable to identify a balloon with a nuclear warhead from the balloon bait.

\section{Conclusion}

Firstly, the thermal energy of warhead target/decoy is analyzed, and then the surface temperature field of warhead target is calculated by panel partition method. Finally, the infrared radiation characteristics of warhead target and balloon decoy are analyzed. From the above analysis, it can be seen that the thermal characteristics of balloon decoy and balloon decoy with warhead after release are not. Similarly, if the measures are not proper, the infrared detector will easily distinguish the target. According to the analysis results, the penetration measures for two days and nights are put forward.

\section{Conflicts of Interest}

The authors declare no conflicts of interest regarding the publication of this paper.

\section{References}

[1] Huang, P.K. and Runjin (2010) Recognition and Anti-Recognition Technology in Ballistic Missile Countermeasure. Space Electronic Countermeasure, 26, 11-14.

[2] (2009) Analysis of Infrared Photoelectric Identification Technology in the Ballistic Missile Defense System. Infrared and laser engineering, 38, 759-766. 
[3] (2007) Image Surface Illuminance Model of Space-Based Infrared Detection with Ballistic Missile Booster and Its Application. Journal of Infrared and Millimeter Wave, 26, 425-428.

[4] River, F.S. and Nie, W.S. (2006) Midcourse Ballistic Missile and Background Infrared Radiation Research Survey. Infrared and Laser Engineering, 27, 19-22.

[5] Zhu, L.L. and Zhou, N. (2007) Brief Introduction of Airborne Infrared Measurement System and Photoelectric Test Facility of U.S. Air Force and Navy. Reference for Management and Technical Research of Foreign Military Target and Environmental Characteristics, 11.

[6] Peng, D. (2006) NASA Space Shuttle Infrared Background Characteristic Measurement Test. Reference for Foreign Military Target and Environmental Characteristic Management and Technical Research, 01.

[7] Xu, X.J. (2006) Overview of the US Mid-Range Space Experiments (MSX) Program. References for Foreign Military Objectives and Environmental Characteristics Management and Technical Research, 08. 